\title{
Tópicos Emergentes em Insuficiência Cardíaca: COVID-19 e Insuficiência Cardíaca
}

\author{
Emerging Topics in Heart Failure: COVID-19 and Heart Failure
}

\author{
Livia Adams Goldraich, ${ }^{\circledR}$ Odilson Marcos Silvestre, ${ }^{2 @}$ Edval Gomes, ${ }^{3}{ }^{\circledR}$ Bruno Biselli, ${ }^{4}{ }^{\oplus}$ Marcelo Westerlund Montera ${ }^{5}$ \\ Hospital de Clínicas de Porto Alegre, ${ }^{1}$ Porto Alegre, RS - Brasil \\ Universidade Federal do Acre, ${ }^{2}$ Rio Branco, AC - Brasil \\ Universidade Estadual de Feira de Santana, ${ }^{3}$ Feira de Santana, BA - Brasil \\ Instituto do Coração da Universidade de São Paulo, ${ }^{4}$ São Paulo, SP - Brasil \\ Hospital Pró-Cardíaco, ${ }^{5}$ Rio de Janeiro, RJ - Brasil \\ Carta científica referente ao Heart Failure Summit Brazil 2020 / Departamento de Insuficiência Cardíaca - DEIC/SBC
}

\section{Introdução}

Alguns autores têm proposto a denominação de "síndrome cardiovascular aguda pela COVID-19" para descrever as alterações do sistema cardiovascular associadas à infecção pelo SARS-CoV-2. ${ }^{1}$ Entre as diferentes manifestações, podem ocorrer injúria miocárdica, miocardite, infarto do miocárdio com coronárias normais (MINOCA), arritmias, Takotsubo, derrame pericárdico, insuficiência cardíaca (IC), além dos fenômenos tromboembólicos ${ }^{2,3}$ (Tabela 1). Enfatizaremos a injúria miocárdica, a miocardite, a síndrome Takotsubo e a ocorrência e peculiaridades da COVID-19 em portadores de IC.

\section{Injúria Miocárdica}

O impacto da injúria miocárdica relacionada à infecção por SARS-CoV-2 foi reconhecido já no início da pandemia, com dados chineses e, subsequentemente, múltiplas coortes de diversos países, invariavelmente demonstrando aumento de mortalidade associado à elevação de troponina. ${ }^{2,4}$ Os potenciais mecanismos de envolvimento cardíaco em pacientes com COVID-19 são múltiplos e envolvem fatores diretos de infecção viral e, principalmente, indiretos de dano miocárdico. A presença do receptor da enzima de conversão da angiotensina 2 na superfície do cardiomiócito e das células endoteliais vasculares sugeria que o SARS-CoV-2 poderia levar ao dano tóxico pelo vírus e, consequentemente, miocardite. ${ }^{5}$ No entanto, um estudo alemão baseado em autópsias detectou cópias do vírus em células intersticiais e em macrófagos invadindo o miocárdio, mas não em cardiomiócitos. ${ }^{6}$ Além disso, a presença de genoma viral no coração não se associou a infiltrado inflamatório típico

\section{Palavras-chave}

COVID-19; Insuficiência Cardíaca; Miocardite; Takotsubo; Lesão Miocárdica.

\section{Correspondência: Odilson Marcos Silvestre •}

Rua Quintino Bocaiuva, 1108. CEP 69900-785. José Augusto, Rio Branco, AC - Brasil

E-mail: odilsonsilvestre@yahoo.com.br

Artigo recebido em 06/10/2020, revisado em 06/10/2020, aceito em 06/10/2020

DOI: https://doi.org/10.36660/abc.20201081 de miocardite, sugerindo que a infecção pelo SARS-CoV-2 não ocasiona um quadro celular inflamatório clássico. É possível que outras vias de injúria inflamatória possam desempenhar papel no dano miocárdico pelo vírus, envolvendo, particularmente, vasculite e ativação sistêmica da liberação de citocinas.

\section{Miocardite}

Apesar desses dilemas em relação à fisiopatologia do dano miocárdico causado pela infecção por COVID-19, diversos casos de miocardite fulminante vêm sendo reportados. ${ }^{5,7}$ As apresentações clínicas parecem ser variáveis, porém semelhantes às manifestações de miocardites causadas por outros agentes virais, incluindo dor torácica, dispneia, arritmias, febre e algum grau de disfunção ventricular. $\mathrm{O}$ eletrocardiograma pode demonstrar alterações difusas do segmento ST, infra ou supradesnível de segmentos PR ou ST e, às vezes, pode mimetizar alterações compatíveis com infarto agudo com supradesnível de ST.2,3 As troponinas encontramse elevadas, embora em valores absolutos menores do que os observados em síndromes coronarianas. A dosagem de peptídeos natriuréticos pode auxiliar na confirmação diagnóstica de miocardite, particularmente quando os níveis de troponina estiverem pouco alterados. Alterações difusas na motilidade ao ecocardiograma parecem ser mais comuns na suspeita de miocardite, comparativamente às síndromes isquêmicas agudas. O uso da ressonância magnética pode auxiliar na confirmação diagnóstica, apresentando padrão típico de comprometimento inflamatório. Parece haver uma alta proporção de pacientes com elevação de troponina e/ou alterações de eletrocardiograma que demonstram persistência de quadro inflamatório e/ou achados de miocardite subclínica na ressonância magnética cardíaca, mesmo após a recuperação da COVID-19 (aproximadamente 56 dias após quadro agudo). ${ }^{8}$

A concomitância de níveis elevados de troponinas, alterações no eletrocardiograma e presença de algum grau de disfunção ventricular configuram pior prognóstico na miocardite por SARS-CoV-2, embora qualquer evidência de injúria miocárdica deva ser considerada como marcador de risco para pacientes com diagnóstico de COVID-19, independentemente da suspeita de miocardite. Assim como no manejo da infecção por COVID-19 e suas 
Tabela 1 - Espectro das manifestações cardiovasculares pela COVID-19

\begin{tabular}{|c|c|}
\hline Fenótipo & Características importantes \\
\hline Injúria miocárdica & Troponina elevada ( > percentil 99) \\
\hline Síndrome coronária aguda & $\begin{array}{c}\text { Troponina elevada ( > percentil } 99 \text { ) + sintomas + } \\
\text { doença coronária obstrutiva }\end{array}$ \\
\hline MINOCA & $\begin{array}{l}\text { Troponina elevada ( > percentil 99) + sintomas } \\
\text { (sem doença coronária obstrutiva) }\end{array}$ \\
\hline $\begin{array}{l}\text { Miocardite ou } \\
\text { miopericardite }\end{array}$ & $\begin{array}{c}\text { Troponina elevada ( > percentil 99) + sintomas + } \\
\text { achados compatíveis na BEM ou RNM }\end{array}$ \\
\hline Takotsubo & $\begin{array}{c}\text { Troponina elevada ( > percentil 99) + sintomas + } \\
\text { ventriculografia típica }\end{array}$ \\
\hline Arritmias & $\begin{array}{l}\text { Taquiarritmias atriais, ventriculares } \\
\text { ou bradiarritmias }\end{array}$ \\
\hline Insuficiência cardíaca & $\begin{array}{l}\text { IC crônica descompensada por COVID-19 ou } \\
\text { IC aguda causada por um dos seguintes: SCA, } \\
\text { MINOCA, miocardite ou Takotsubo }\end{array}$ \\
\hline Derrame pericárdico & Muitas vezes associada à (mio)pericardite \\
\hline Tromboembolismo & TVP, TEP, AVC ou embolia periférica \\
\hline \multicolumn{2}{|c|}{$\begin{array}{l}\text { MINOCA: infarto do miocárdio com coronárias normais; BEM: biópsia } \\
\text { endomiocárdica; RNM: ressonância magnética; IC: insuficiência cardiaca; } \\
\text { SCA: síndrome coronária aguda; TVP: trombose venosa profunda; TEP: } \\
\text { tromboembolismo pulmonar; AVC: acidente vascular cerebral. }\end{array}$} \\
\hline
\end{tabular}

múltiplas repercussões sistêmicas, a estratégia terapêutica específica para miocardite por SARS-CoV-2 baseia-se principalmente em tratamento de suporte sistêmico. O uso de imunomoduladores, como corticosteroides, e bloqueadores do receptor de interleucina 6, como tocilizumab, foi reportado em relatos de caso e em revisão sistemática recente e seus efeitos na COVID-19 ainda estão sob investigação. ${ }^{9,10}$ Arritmias merecem algum grau de monitorização, embora não haja tratamento específico recomendado no contexto da infecção por SARS-CoV-2. ${ }^{11}$

\section{Síndrome Takotsubo}

Houve um aumento de cerca de cinco vezes na incidência de síndrome Takotsubo durante a pandemia por SARS-CoV-2, em comparação com as síndromes coronárias agudas, que tiveram redução dos casos no mesmo período. Uma coorte da Cleveland Clinic mostrou que, durante a pandemia, cerca de $8 \%$ dos casos que se apresentaram como síndrome coronária aguda eram Takotsubo, diferente da incidência de $1 \%$ relatada antes da pandemia. ${ }^{12}$ Os mecanismos fisiopatogênicos associados a esse aumento podem ser resultado direto do vírus, causando uma miocardite que mimetiza o Takotsubo (cardiomiopatia Takotsubo-like) ou, mais provável, o efeito do estresse psicológico imposto pela quarentena e pelo risco da pandemia, pela perda da interação social causada pelas regras de distanciamento e pelas consequências socioeconômicas da pandemia. As séries de caso mostram apresentação clínica semelhante àquelas relacionadas a outros fatores estressantes e mortalidade similar àquela descrita fora da situação de pandemia por COVID-19.

\section{COVID-19 em Pacientes com IC}

A presença de IC no contexto da COVID-19 identifica um subgrupo de manejo complexo e de maior morbimortalidade. A IC pode representar tanto um fator de risco para uma pior evolução infecciosa quanto uma complicação cardiovascular grave causada pelo vírus SARS-CoV-2. ${ }^{13}$ A ativação da cascata inflamatória, a hiperestimulação do sistema neuro-humoral e a toxicidade viral direta representam alguns dos possíveis mecanismos fisiopatológicos para a IC aguda nova ou descompensada nesse cenário.

Pacientes hospitalizados com IC devem, preferencialmente, realizar pesquisa de SARS-CoV-2 em virtude da sobreposição de sinais e de sintomas e devem ser submetidos à avaliação minuciosa sobre o status volêmico, além de avaliação laboratorial, ecocardiográfica e radiológica. A COVID-19 pode ser entendida como uma síndrome inflamatória sistêmica e essa característica deve ser considerada na prescrição de vasodilatadores para indivíduos com IC aguda. A manutenção de medicações recomendadas pelas diretrizes deve ocorrer durante a internação naqueles com hemodinâmica preservada e normotensos. Outras estratégias, como a telemedicina, incluindo o telemonitoramento e as consultas virtuais, foram importantes no manejo da IC crônica e na prevenção infecciosa. Além de reduzir o risco de exposição viral, esses programas auxiliaram nas orientações preventivas para a COVID-19 e na identificação de pacientes sob risco de descompensação. ${ }^{14}$

\section{Considerações Finais}

O espectro do acometimento cardíaco pela COVID-19 em pacientes com ou sem insuficiência cardíaca prévia é atualmente um conhecimento em evolução. Da mesma forma, as consequências a médio e longo prazo dos efeitos da infecção por SARS-CoV-2 no coração poderão trazer desdobramentos clínico-epidemiológicos relevantes, porém ainda pouco previsíveis. É provocativo considerar que possamos estar diante de uma nova etiologia de cardiomiopatia, que eventualmente poderá contribuir para o aumento da incidência de IC nos próximos anos.

Lista de participantes do Heart Failure Summit Brazil 2020 / Departamento de Insuficiência Cardíaca - DEIC/SBC

Aguinaldo Freitas Junior, Andréia Biolo, Antonio Carlos Pereira Barretto, Antônio Lagoeiro Jorge, Bruno Biselli, Carlos Eduardo Montenegro, Denilson Campos de Albuquerque, Dirceu Rodrigues de Almeida, Edimar Alcides Bocchi, Edval Gomes dos Santos Júnior, Estêvão Lanna Figueiredo, Evandro Tinoco Mesquita, Fabiana G. Marcondes-Braga, Fábio Fernandes, Fabio Serra Silveira, Felix José Alvarez Ramires, Fernando Atik, Fernando Bacal, Flávio de Souza Brito, Germano Emilio Conceição Souza, Gustavo Calado de Aguiar Ribeiro, Humberto Villacorta Jr., Jefferson Luis Vieira, João David de Souza Neto, João Manoel Rossi Neto, José Albuquerque de Figueiredo Neto, Lídia Ana Zytynski Moura, Livia Adams Goldraich, Luís Beck-da-Silva, Luís Eduardo Paim Rohde, Luiz Claudio Danzmann, Manoel Fernandes Canesin, Marcelo Bittencourt, Marcelo Westerlund Montera, Marcely Gimenes Bonatto, Marcus Vinicius Simões, Maria da Consolação Vieira Moreira, Miguel Morita Fernandes da Silva, Monica Samuel Avila, Mucio Tavares de Oliveira Junior, 
Nadine Clausell, Odilson Marcos Silvestre, Otavio Rizzi Coelho Filho, Pedro Vellosa Schwartzmann, Reinaldo Bulgarelli Bestetti, Ricardo Mourilhe Rocha, Sabrina Bernadez Pereira, Salvador Rassi, Sandrigo Mangini, Silvia Marinho Martins, Silvia Moreira Ayub Ferreira, Victor Sarli Issa.

\section{Contribuição dos autores}

Concepção e desenho da pesquisa, Obtenção de dados, Redação do manuscrito e Revisão crítica do manuscrito quanto ao conteúdo intelectual importante: Goldraich LA, Silvestre OM, Gomes E, Biselli B, Montera MW

\section{Referências}

1. Hendren NS, Drazner MH, Bozkurt B, Cooper LT. Description and Proposed Management of the Acute COVID-19 Cardiovascular Syndrome. Circulation. 2020;141:1903-1914

2. Costa IBSS, Bittar CS, Rizk SI, Araújo Filho AE, Santos KAQ, Machado TIV, et al. O Coração e a COVID-19: O que o Cardiologista Precisa Saber. Arq. Bras. Cardiol, 2020 114: 805-816.

3. Figueiredo Neto JA, Marcondes-Braga FG, Moura LZ, Figueiredo AMS, Figueiredo VMS, Mourilhe-Rocha R, et al. Coronavirus Disease 2019 and the Myocardium. Arq Bras Cardiol. 2020;114(6):1051-1057.

4. Shi S, Qin M, Shen B, Cai Y, Liu T, Yang F, et al. Association of Cardiac Injury With Mortality in Hospitalized Patients With COVID-19 in Wuhan, China. JAMA Cardiol. 2020 Jul1;5(7):802-810.

5. Inciardi RM, Lupi L, Zaccone G, Italia L, Raffo M, Tomasoni D, et al. Cardiac involvement in a patient with coronavirus disease 2019 (COVID-19). JAMA Cardiol 2020 Jul 1;5(7):819-824.

6. Lindner D, FitzekA, Bräuninger H, Aleshcheva G, Edler C, Meissner K, et al. Association of Cardiac Infection With SARS-CoV-2 in Confirmed COVID-19 Autopsy Cases. JAMA Cardiol 2020;e203551.

7. Hu H, Ma F, Wei X, Fang Y. Coronavirus fulminant myocarditis treated with glucocorticoid and human immunoglobulin. Eur Heart J 2020 Mar 16; ehaa190.

8. Ng M-Y, Ferreira VM, Leung ST, Lee JCY, Fong AHT, Liu RWT, et al. Recovered COVID-19 Patients Show Ongoing Subclinical Myocarditis as Revealed by

\section{Potencial conflito de interesses}

Declaro não haver conflito de interesses pertinentes.

\section{Fontes de financiamento}

O presente estudo não teve fontes de financiamento externas.

\section{Vinculação acadêmica}

Não há vinculação deste estudo a programas de pósgraduação.

Cardiac Magnetic Resonance Imaging. JACC: Cardiovasc Imaging 2020, doi: https://doi.org/10.1016/j.jcmg.2020.08.012

9. Siripanthong B, Nazarian S, Muser D, Deo R, Santangeli P, Khanji MY, et al Recognizing COVID-19-related myocarditis: The possible pathophysiology and proposed guideline for diagnosis and management. Heart Rhythm 2020;17:1463-1471

10. Coyle J, Igbinomwanhia E, Sanchez-Nadales A, Danciu S, Chu C, Shah N, et al. A recovered case of COVID-19 myocarditis and ARDS treated with corticosteroids, tocilizumab, and experimental AT-001. JACC Case Rep 2020 Jul 15;2(9):1331-1336.

11. Kariyanna PT, Sutarjono B, Grewal E, Singh KP, Aurora L, Smith L, et al. A Systematic Review of COVID-19 and Myocarditis. Am J Med Case Rep. 2020; 8(9):299-305.

12. Jabri A, Kalra A, Kumar A, Alameh A, Adroja S, Bashir H, et al. Incidence of Stress Cardiomyopathy During the Coronavirus Disease 2019 Pandemic. JAMA Network Open. 2020;3(7):e2014780.

13. Yonas E, Alwi I, Pranata R, Huang I, Lim MA, Gutierrez EJ, et al. Effect of heart failure on the outcome of COVID-19 - A meta analysis and systematic review. Am J Emerg Med. 2020;doi:10.1016/j.ajem.2020.07.009.

14. Salzano A, D'Assante R, Stagnaro FM, Valente V, Crisci G, Giardino F, et al. Heart failure management during the COVID-19 outbreak in Italy: a telemedicine experience from a heart failure university tertiary referral centre. Eur J Heart Fail. 2020;22(6):1048-1050. doi:10.1002/ejhf.1911 